\title{
Colorectal surgery surgical site infection prevention practices in British Columbia
}

\author{
Amandeep Ghuman, MD, MPH \\ Ahmer A. Karimuddin, MD \\ Carl J. Brown, MD \\ Manoj J. Raval, MD \\ P. Terry Phang, MD
}

Accepted Sept. 18, 2020

\section{Correspondence to: \\ P.T. Phang \\ St. Paul's Hospital \\ 1081 Burrard St \\ Vancouver BC V6Z 1 Y 6 \\ tphang@providencehealth.bc.ca}

Cite as: Can J Surg 2021 October 1; 64(5). doi: 10.1503/cjs.007220

\section{SUMmARY}

Surgical site infections (SSI) pose significant morbidity after colorectal surgery. We sought to document current practices in colorectal surgery SSI prevention in British Columbia (BC). Reporting the current provincial landscape on SSI prevention helps to understand the foundation upon which improvements can take place. We surveyed all BC surgeons performing elective colon and rectal resections, and 97 surveys were completed (60\% response rate). Eighty-six per cent of respondent hospitals tracked SSI rates. The reported superficial SSI was less than $5 \%$ and the anastomotic leak/organ space rate was less than $10 \%$. All respondents gave preoperative prophylactic antibiotics, with $24 \%$ continuing antibiotics postoperatively; $62 \%$ are using oral antibiotics $(\mathrm{OAB})$ and mechanical bowel preparation (MBP) and 29\% use MBP without OAB. Areas for improvement include $\mathrm{OAB}$ with $\mathrm{MBP}$ and discontinuing prophylactic antibiotics postoperatively, as recommended by the World Health Organization.

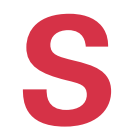

urgical site infections (SSIs) cause significant morbidity after colorectal surgery; rates reported in the literature range from $1 \%$ to $45 \% .{ }^{1}$ Modifiable SSI risk factors for colorectal surgery have been identified, ${ }^{2}$ and prevention strategies have been implemented into perioperative interventions, and have been shown to significantly reduce SSI rates. ${ }^{3}$ The World Health Organization (WHO) published guidelines for SSI prevention, ${ }^{4}$ but it is unknown whether these guidelines are followed in Canada and, specifically, in British Columbia (BC).

We designed a survey to document whether SSIs are being monitored, identify current SSI rates, and determine what strategies BC surgeons use for SSI prevention in elective colorectal surgery.

\section{SURVEY DESIGN}

All BC surgeons performing elective colon and rectal resections were invited to participate. Survey questions were based on WHO SSI guidelines as well as input from colorectal surgeons and a Harvard University Survey Research Methods expert (Dr. Thomas W. Mangione, Harvard University, Cambridge, Mass.: personal communication, 2017). Twenty-three questions were formulated to assess whether SSIs are monitored in BC hospitals, surgeon reported SSI rates, and use of SSI prevention measures (preoperative, intraoperative, postoperative). The online survey link was distributed on the Surgeons of BC listserv with anonymous, voluntary participation.

\section{OUtcomes}

Ninety-seven surveys were returned, for a $60 \%$ response rate. Demographic location of respondents is shown in Figure 1: $81 \%$ were from teaching hospitals (17\% academic, $64 \%$ community), $7 \%$ from community nonteaching hospitals and $12 \%$ from rural. Eighty-six per cent had a 


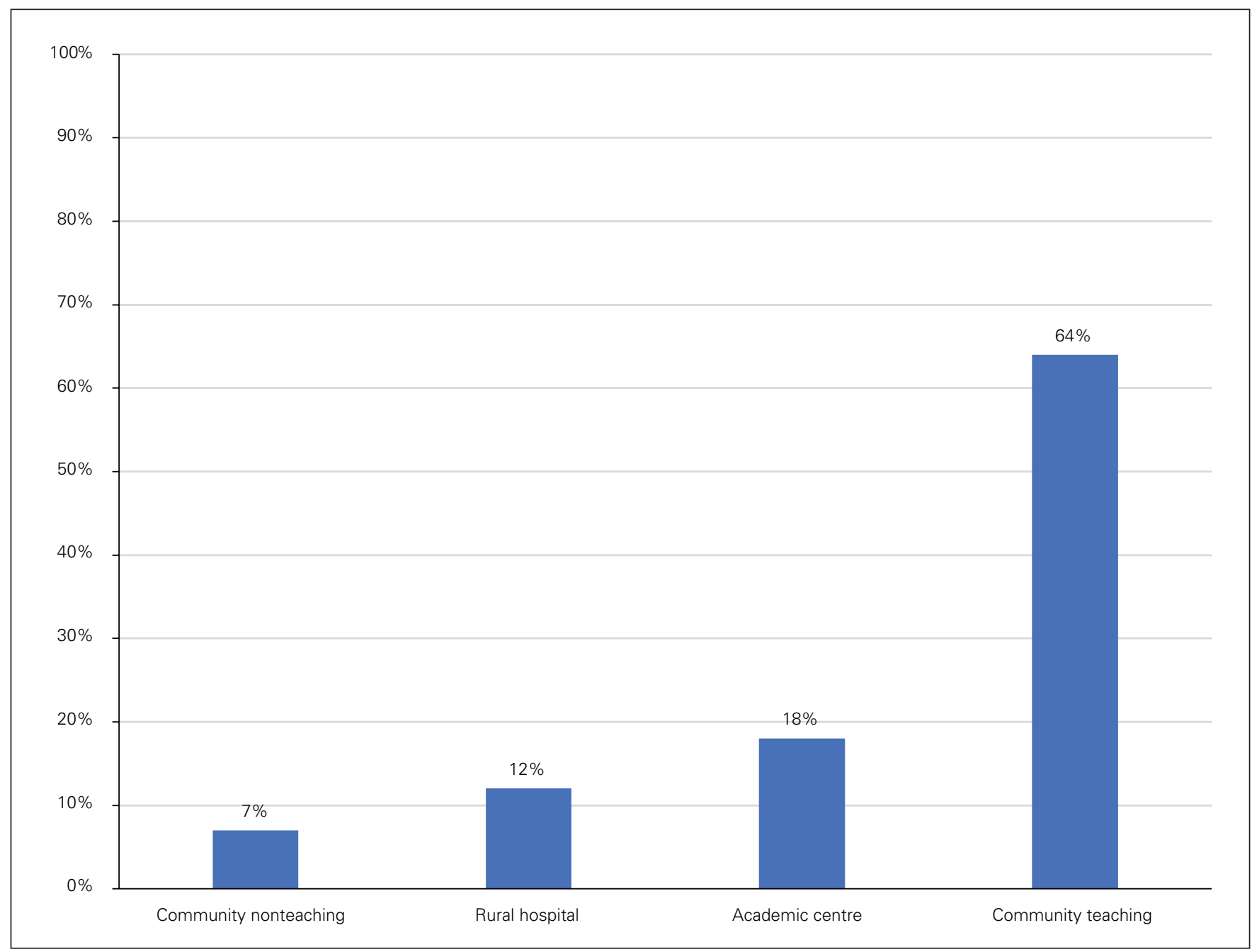

Fig. 1. Demographic distribution of survey respondents.

hospital SSI tracking system, while 14\% did not. Seventy-six per cent reported that their superficial SSI rate was $10 \%$ or less, and $79 \%$ said the anastomotic leak/organ space SSI rate was $5 \%$ or less (Figure 2).

\section{Preoperative practices}

Ninety-two per cent of survey respondents provide the following preoperative counselling: smoking cessation $(83 \%)$, nutritional support $(67 \%)$, tight glycemic control (55\%), weight loss (8\%), and physical activity (8\%). For skin cleansing at home the night before surgery, $33 \%$ recommend chlorhexidine wipes/ sponge, $25 \%$ antimicrobial soap, $20 \%$ plain soap, and $23 \%$ nothing.

For bowel preparation, $62 \%$ prescribe mechanical bowel preparation (MBP) with oral antibiotics (OAB), $29 \% \mathrm{MBP}$ alone, 4\% OAB alone, and 5\% neither MBP nor OAB (Figure 3). All respondents reported they administer prophylactic intravenous antibiotics within
60 minutes of skin incision: $93 \%$ cephalexin and metronidazole, $4 \%$ cephalexin only, and 3\% ceftriaxone and metronidazole. For operative site hair removal, $95 \%$ use hair clippers, $4 \%$ shave, and $1 \%$ do not routinely remove hair. For abdominal skin preparation, 98\% use alcohol-based chlorhexidine, and $2 \%$ use povidonebased preparation.

\section{Intraoperative practices}

Colorectal procedures are done laparoscopically by $48 \%$ in $76 \%-100 \%$ of cases, by $24 \%$ in $51 \%-75 \%$ of cases, by $15 \%$ in $26 \%-50 \%$ of cases, by $13 \%$ in $1 \%-25 \%$ of cases, and by $1 \%$ in no cases (Figure 4).

Regarding use of fraction of inspired oxygen $\left(\mathrm{FiO}_{2}\right)$ of $80 \%$ intraoperatively and 2 hours postoperatively, $56 \%$ of respondents were unaware of this recommendation, $21 \%$ followed this practice, and $24 \%$ did not. Sixty-two per cent reported the existence of glucose control protocols at their institutions, $28 \%$ said there were none, and $10 \%$ 


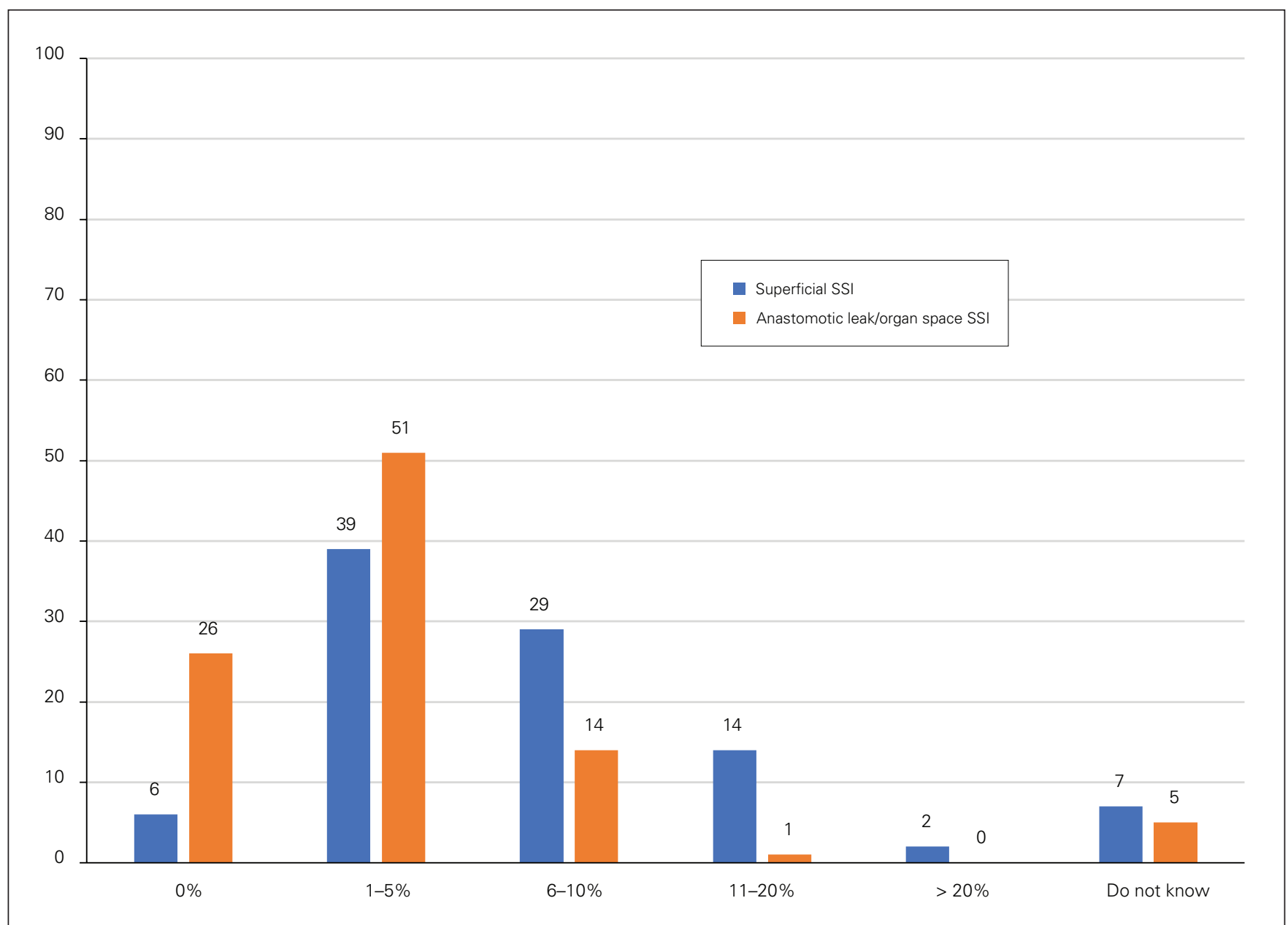

Fig. 2. Survey responses to the question, "Considering resections you personally have done over the past year, what proportion develop superficial wound infections (blue bar) and what proportion develop anastomotic leak/organ space infection (orange bar)?"

did not know of any such protocols. Seventy-nine per cent reported using goal-directed fluid therapy, $16 \%$ did not, and 5\% were not aware of the recommendation to do so. All respondents reported use of warming devices to maintain patient normothermia in the operating room: $71 \%$ always, $26 \%$ most of the time, $2 \%$ half the time, and $1 \%$ sometimes. A wound protector was used by $37 \%$ always, $28 \%$ most of the time, $6 \%$ about half the time, $10 \%$ sometimes, and $19 \%$ never.

\section{Postoperative practices}

Prophylactic negative pressure wound dressings are never used by $46 \%$ of respondents, by $45 \%$ sometimes, by $2 \%$ about half the time, by $3 \%$ most of the time, and by $3 \%$ always. All respondents use an Enhanced Recovery After Surgery or similar multidisciplinary protocol postoperatively: $68 \%$ always, $25 \%$ most of the time, $4 \%$ about half the time, and 3\% sometimes. Seventy-six per cent reported they do not administer postoperative prophylactic antibiotics; however, $19 \%$ administer antibiotics for 24 hours, and 5\% for more than 24 hours.

\section{Discussion}

To our knowledge, this is the first Canadian survey on colorectal surgery SSI prevention measures. Eighty per cent of survey respondents described their hospital as a teaching facility. This distribution is in keeping with the majority of $\mathrm{BC}$ hospitals (77\%) being training sites associated with the University of British Columbia general surgery residency program. It is important to understand our current landscape of practice to make quality improvements (QI).

Most surgeons follow WHO guidelines,${ }^{4}$ including administering preoperative prophylactic antibiotics within 60 minutes of skin incision (100\%), removing hair with clippers (96\%), and preparing skin with alcohol-based antiseptics (98\%). Strong evidence-based WHO guidance to use $\mathrm{MBP}$ in combination with $\mathrm{OAB}$ in bowel preparation was not followed by the $29 \%$ using MBP alone and the $24 \%$ using prophylactic antibiotics alone postoperatively, and therefore improvement is possible. Compliance with conditional WHO recommendations are more varied, as evidence is moderate to low for those practices. 


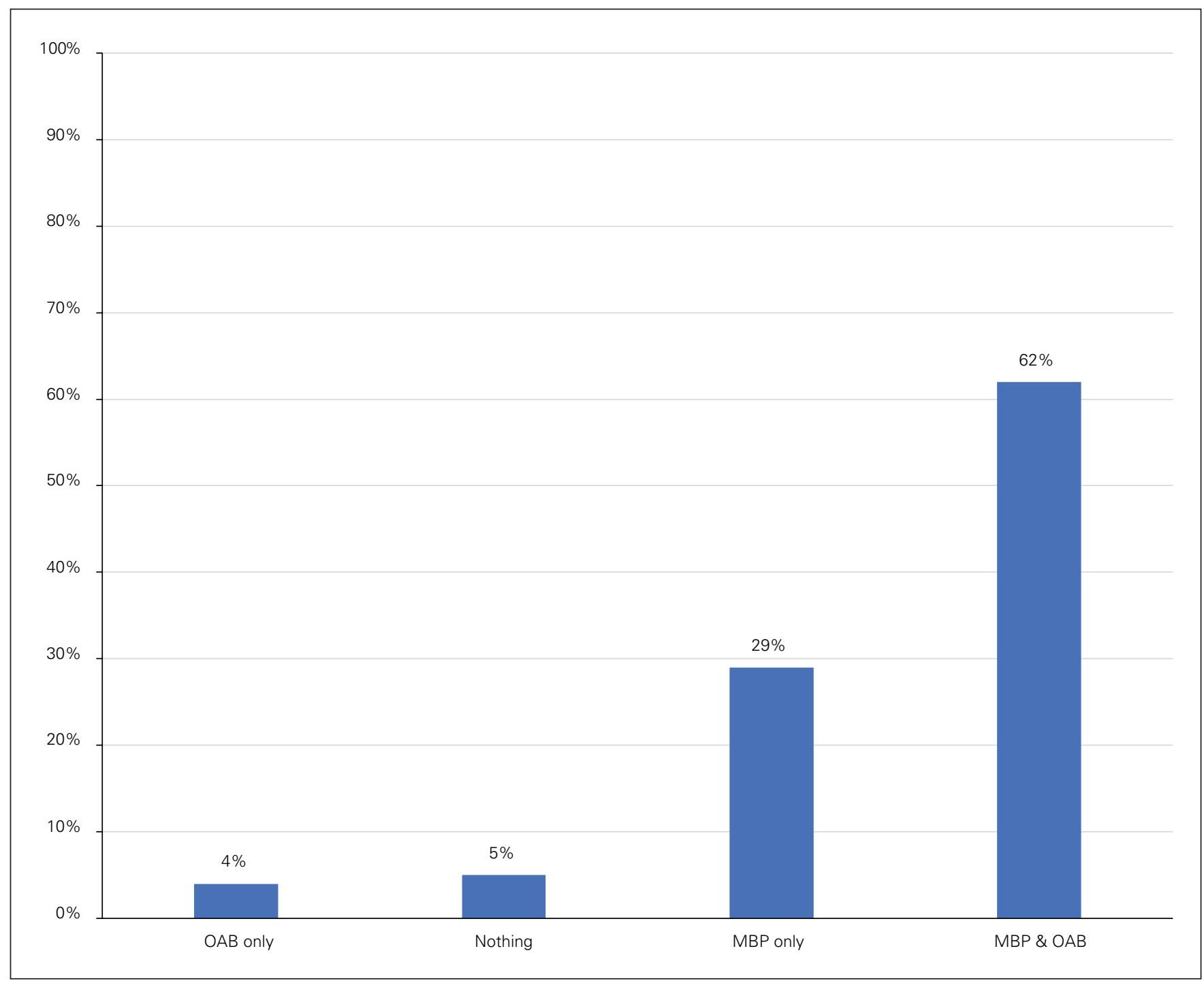

Fig. 3. Survey responses to the question, "When thinking about bowel preparation, which of the following do you most often recommend to your patients?" MBP = mechanical bowel preparation; $\mathrm{OAB}=$ oral antibiotics.

Meta-analysis of 23 studies including 17557 patients found significant reduction in overall, superficial and organ/space SSIs. ${ }^{1}$ The greatest SSI reduction resulted from "care bundles" that included MBP with $\mathrm{OAB}$, use of a sterile instrument closure tray and glove change before closure. ${ }^{1}$ However, compliance with individual components varied widely $(19 \%-$ 99\%). ${ }^{1}$ Moving forward, a compliance measurement strategy is necessary to implement a province-wide program.

The Illinois Surgical Quality Improvement Collaborative demonstrated successful implementation of a multifaceted SSI reduction bundle across 32 hospitals. ${ }^{5}$ Their strategy entailed guided implementation, data monitoring, a central coordinating centre providing telephone/email support, and a comprehensive Colorectal SSI Reduction Toolkit. ${ }^{5}$ By following an established QI structure they showed that higher compliance rates translated into lower SSI rates. ${ }^{5}$ Using these techniques across our varied landscape may prove as beneficial in reducing our SSIs.
With programs such as the American College of Surgeons National Surgical Quality Improvement Program (ACS-NSQIP), surgeons are capable of tracking their SSI rates and improving surgical care through use of riskadjusted clinical data. There are currently 95 ACS-NSQIP hospitals across Canada, 23 of which are in BC. Although this represents $59 \%$ of $\mathrm{BC}$ hospitals, $86 \%$ of surveyors had SSI tracking programs. Further information regarding the nature of these programs is needed before adoption of a standardized province-wide program and implementing QI initiatives for colorectal surgery SSI reduction.

\section{Conclusion}

The majority of BC surgeons surveyed had an established SSI tracking program and reported low SSI rates. A majority followed WHO recommendations of prophylactic preoperative antibiotics, use of hair clippers and 


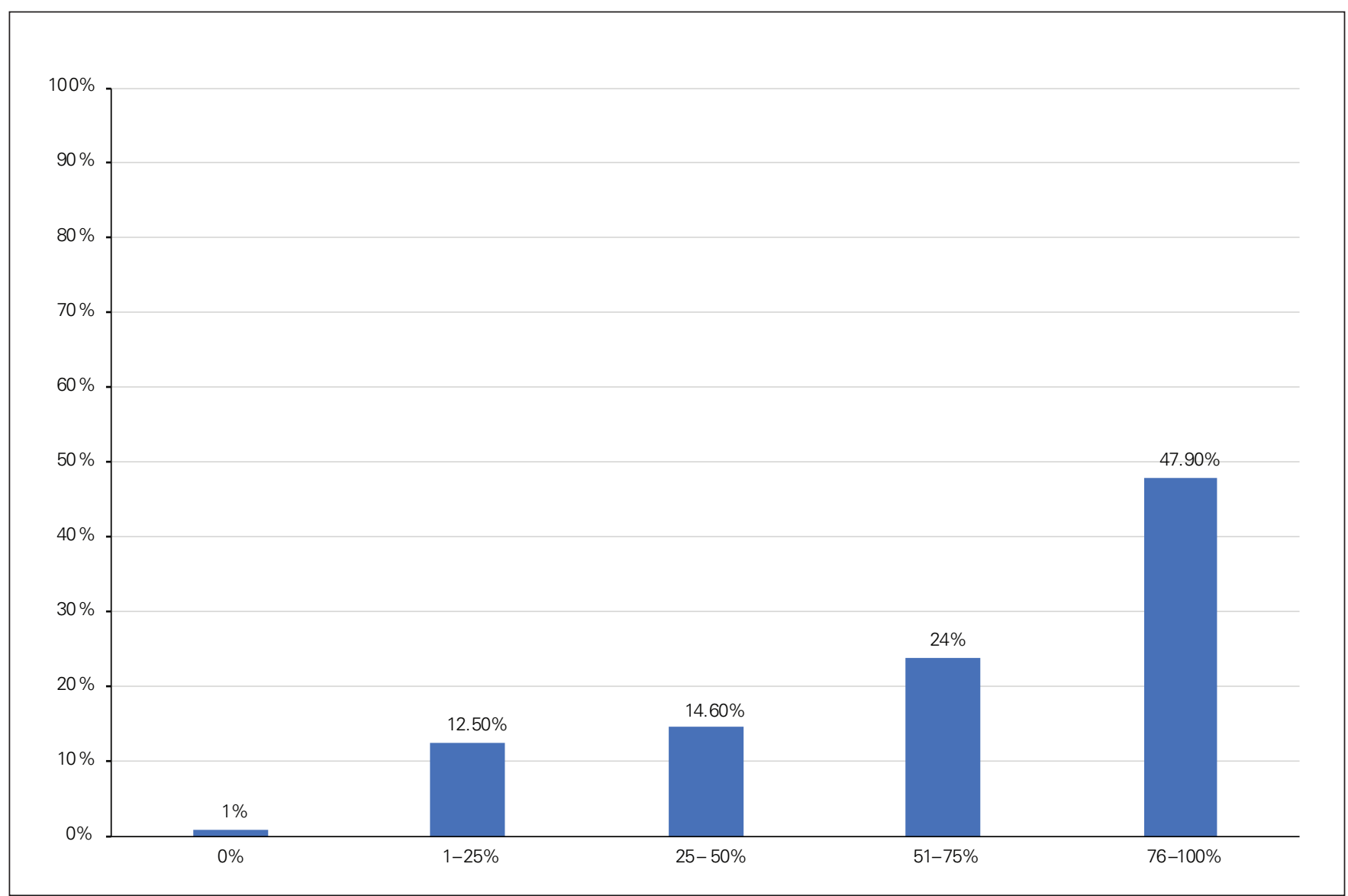

Fig. 4. Percentage of colorectal cases performed laparoscopically in the past year.

alcohol-based chlorohexidine skin preparation. However, improvement is possible with standardized $\mathrm{OAB}$ with MBP and discontinuing postoperative prophylactic antibiotics. A province-wide SSI reduction program is possible through wide multidisciplinary collaboration using SSI tracking and iterative changes.

Affiliations: From the Department of Surgery, Division of General Surgery, University of British Columbia, Vancouver, BC (Ghuman); and the Department of Surgery, St. Paul's Hospital, Vancouver, BC (Karimuddin, Brown, Raval, Phang).

Competing interests: None declared.

Contributors: All authors contributed substantially to the conception, writing and revision of this article and approved the final version for publication.

Content licence: This is an Open Access article distributed in accordance with the terms of the Creative Commons Attribution (CC BYNC-ND 4.0) licence, which permits use, distribution and reproduction in any medium, provided that the original publication is properly cited, the use is noncommercial (i.e., research or educational use), and no modifications or adaptations are made. See: https://creativecommons.org/ licenses/by-nc-nd/4.0/

\section{References}

1. Zywot A, Lau CSM, Fletcher HS, et al. Bundles prevent surgical site infections after colorectal surgery: meta-analysis and systematic review. 7 Gastrointest Surg 2017;21:1915-30.

2. Young H, Knepper B, Moore EE, et al. Surgical site infection after colon surgery: national healthcare safety network risk factors and modeled rates compared with published risk factors and rates. 7 Am Coll Surg 2012;214:852-9.

3. Weiser MR, Gonen M, Usiak S, et al. Effectiveness of a multidisciplinary patient care bundle for reducing surgical-site infections. Br7 Surg 2018;105:1680-7.

4. World Health Organization. Global Guidelines for the Prevention of Surgical Site Infection. Geneva: WHO;2016. Available: http://www. who.int (accessed 2019 Oct. 21).

5. McGee MF, Kreutzer L, Quinn CM, et al. Leveraging a comprehensive program to implement a colorectal surgical site infection reduction bundle in a statewide quality improvement collaborative. Ann Surg 2019;270:701-11. 